\title{
Perfil epidemiológico dos pacientes com hipertensão arterial sistêmica cadastrados na Casa Saúde da Família Águas Lindas II, Belém, PA*
}

\author{
Epidemiological profile of patients with systemic \\ arterial hypertension, registered in the Casa Saúde \\ da Família Águas Lindas II, Belém - PA
}

\section{Everton Mesquita Dias ${ }^{1}$, Roose Mary Nunes Pereira², Herlândia Soares Cavalcante ${ }^{3}$, Natasha Silva Ramalheiro ${ }^{3}$, Noelle Freire da Silva ${ }^{3}$, Moisés Ribeiro de Carvalho Júnior ${ }^{4}$}

\begin{abstract}
Dias EM, Pereira RMN, Cavalcante HS, Ramalheiro NS, Silva NF, Carvalho Júnior MR. Perfil epidemiológico dos pacientes com hipertensão arterial sistêmica cadastrados na Casa Saúde da
\end{abstract} Família Águas Lindas II, Belém - PA. Rev Med (São Paulo). 2009jul.-dez.;88(3/4):191-8.

RESUMO: OBJETIVO: Identificar o perfil epidemiológico dos pacientes com Hipertensão Arterial Sistêmica (HAS) inscritos no Programa Nacional de Atenção à Hipertensão Arterial e Diabetes Mellitus (HIPERDIA) da Casa Saúde da Família Águas Lindas Il, Belém - PA, no ano de 2007. MÉTODO: Estudo observacional, transversal e descritivo, realizado em um corte de 33 pacientes hipertensos, de 40 a 80 anos, de ambos os sexos, cadastrados no HIPERDIA da referida unidade de saúde e selecionados aleatoriamente, por meio de sorteio baseado nos números de matrícula. Aplicou-se um questionário acerca de características biossociais e hábitos de vida. Avaliou-se a pressão arterial (esfigmomanômetro aneróide Diasyst ${ }^{\circledR}$ e estetoscópio Littmann-Cardiology ${ }^{\circledR}{ }^{\circledR}$ ), o índice de massa corpórea (balança antropométrica com estadiômetro Fillizola ${ }^{\circledR}$ ) e a circunferência da cintura (fita métrica inelástica), por meio de uma ficha de avaliação própria. Procedeu-se com análise estatística descritiva através dos programas Bioestat ${ }^{\circledR} 4.0$ e Microsoft ${ }^{\circledR}$ Excel 2007. RESULTADOS: Observou-se que a maioria (40\%) dos pacientes estava na faixa etária de 61 a 70 anos, sendo $82 \%$ do sexo feminino; predominaram a raça mulata $(46 \%)$, renda salarial de 1 a 2 salários mínimos $(61 \%)$, nível fundamental incompleto (52\%), ocupação "do lar" (55\%); a maioria era não tabagista (94\%), não etilista (85\%), sedentária (64\%), sem antecedente familiar (52\%) nem co-morbidades (61\%); todos usavam medicação hipotensora; os níveis pressóricos estavam controlados na maioria (PAS-55\%; PAD-67\%); elevado percentual de obesidade generalizada e/ou sobrepeso (82\%) e central (homens-50\%; mulheres-93\%). CONCLUSÃO: Houve predominância do sexo feminino; mulatos; baixo nível de escolaridade e de renda salarial; baixa ocorrência de etilismo, tabagismo e prática de exercício físico; baixo índice de co-morbidades e antecedentes familiares; alta ocorrência de sobrepeso e obesidade; além de níveis pressóricos controlados, embora um número significativo tenha apresentado PA descontrolada.

DESCRITORES: Hipertensão/epidemiologia; Pressão arterial; Obesidade.

\footnotetext{
* Trabalho realizado na Casa Saúde da Família Águas Lindas II, em Belém - PA.

1. Graduando de Medicina, monitor do Laboratório de Histologia da Universidade do Estado do Pará (UEPA), estagiário do Hospital Pronto Socorro Municipal Mário Pinotti (HPSM-MP) e Liga Acadêmica Paraense de Cirurgia (LAPAC).

2. Graduanda de Medicina, monitora do Laboratório de Anatomia Humana da Universidade do Estado do Pará (UEPA), estagiária do Hospital Pronto Socorro Municipal Mário Pinotti (HPSM-MP) e Liga Acadêmica Paraense de Cirurgia (LAPAC).

3. Graduandas de Medicina da Universidade do Estado do Pará (UEPA).

4. Médico de Família da Casa Saúde da Família Águas Lindas II e Professor auxiliar I da Faculdade de Medicina da Universidade do Estado do Pará (UEPA).

Endereço para correspondência: Everton Mesquita Dias. Passagem Isabel, 550 - Telégrafo; CEP: 66113-240; Belém - PA. e-mail: ton_emd@yahoo.com.br
} 


\section{INTRODUÇÃO}

A Hipertensão Arterial Sistêmica (HAS) vem se configurando como um sério problema de saúde pública no Brasil e no mundo. Ela é um dos mais importantes fatores de risco para o desenvolvimento de doenças cardiovasculares, acidentes vasculares encefálicos e renais ${ }^{1}$.

A HAS é uma enfermidade crônica, de origem multicausal e multifatorial, caracterizada por níveis tensionais elevados, associados a alterações metabólicas e hormonais e a fenômenos tróficos (hipertrofia cardíaca e vascular). Atualmente, a hipertensão arterial em adultos é definida pela pressão arterial sistólica (PAS) $\geq 140 \mathrm{mmHg}$ e/ou pressão arterial diastólica $(P A D) \geq 90 \mathrm{mmHg}^{2}$.

Os níveis pressóricos elevados, persistentes e descontrolados, levam a graves repercussões clínicas, que incluem: insuficiência visual, hemorragia ocular (ambos em virtude de lesões nos vasos sangüíneos dos olhos); angina; fenômenos ateroscleróticos, tais como infarto agudo do miocárdio, insuficiência cardíaca, hipertrofia ventricular; insuficiência renal (decorrente de lesões nos vasos renais), com possibilidade de consequente perda da função renal; insuficiência circulatória; rompimento de aneurismas; sangramentos espontâneos; acidentes vasculares encefálicos (AVEs), dentre outros ${ }^{3}$.

No Brasil, há aproximadamente 17 milhões de portadores de hipertensão arterial. Sabe-se que $35 \%$ da população que tem 40 anos ou mais é acometido pela doença, e esse número vem aumentando. Seu aparecimento está cada vez mais precoce e estima-se que cerca de $4 \%$ das crianças e adolescentes também sejam portadoras, segundo dados do Ministério da Saúde ${ }^{1}$.

Inquéritos de base populacional realizados em algumas cidades brasileiras mostraram prevalência de hipertensão arterial ( $\geq 140 / 90 \mathrm{mmHg}$ ) de $22,3 \%$ a $43,9 \%$. Em indivíduos idosos da cidade de Bambuí - MG, 61,5\% apresentavam hipertensão arterial. A prevalência global de hipertensão entre homens é de $26,6 \%$ (IC 95\% 26,0-27,2\%) e nas mulheres, 26,1\% (IC 95\% 25,5-26,6\%). Ademais, constatou-se que $75 \%$ dos homens e $65 \%$ das muIheres apresentam hipertensão diretamente atribuível a sobrepeso e obesidade ${ }^{4}$.

Também preocupam os resultados de um estudo nacional que revelou que, em indivíduos adultos, $50,8 \%$ sabiam ser hipertensos, 40,5\% estavam em tratamento e apenas $10,4 \%$ tinham pressão arterial controlada - < 140/90 $\mathrm{mmHg}^{4}$.

Em virtude da elevada taxa de morbidade e mortalidade da doença no país e do alarmante número de desconhecimento dos brasileiros a respeito de seu estado de doença, foi instituído um programa específico de atendimento aos hipertensos nas Unidades de Saúde, incluindo as que compõem o Programa Saúde da Família 5 .

No ano 2000, foi lançado o Plano de Reorganização da Atenção à Hipertensão Arterial Sistêmica e ao Diabetes Mellitus no Brasil, pelo qual diversas ações foram implementadas nos estados e municípios. Também foi criado o Programa Nacional de Atenção à Hipertensão Arterial e Diabetes Mellitus (HIPERDIA), que consiste em um sistema de cadastramento e acompanhamento de hipertensos e diabéticos, o que possibilitou a obtenção de maiores e melhores informações acerca do perfil epidemiológico dos pacientes cadastrados e da evolução de seu quadro hipertensivo ${ }^{6}$.

Com base nas preciosas informações do perfil epidemiológico desses pacientes, podem-se detectar fatores de risco para o desenvolvimento da HAS e doenças cardiovasculares, bem como fatores agravantes ${ }^{7}$.

Tendo em vista o aumento crescente do número de casos de pacientes com hipertensão arterial, dada a sua gravidade e baseado nos achados literários acerca do assunto, o presente estudo visa a descrever as características epidemiológicas dos pacientes com hipertensão arterial cadastrados na Casa Saúde da Família Águas Lindas II, em Belém $-P A$.

\section{OBJETIVO}

Identificar o perfil epidemiológico dos pacientes com Hipertensão Arterial Sistêmica (HAS) inscritos no Programa Nacional de Atenção à Hipertensão Arterial e Diabetes Mellitus (HIPERDIA) da Casa Saúde da Família Águas Lindas II, Belém - PA, no ano de 2007.

\section{MÉTODO}

Todos os pacientes desta pesquisa foram estudados segundo os preceitos da Declaração de Helsinque e do Código de Nuremberg e respeitadas as Normas de Pesquisa Envolvendo Seres Humanos (Res. CNS 196/96) do Conselho Nacional de Saúde, após a aprovação pelo Comitê de Ética da UEPA (protocolo 80/07), e autorizado pelos pacientes em estudo ou pelos responsáveis dos mesmos, por meio do Termo de Consentimento Livre e Esclarecido (TCLE).

O estudo realizado foi do tipo observacional, transversal e descritivo, efetuado no período de setembro a novembro de 2007 na Casa Saúde da Família Águas Lindas II. Esta é uma unidade que 
Dias EM, et al. Perfil epidemiológico dos pacientes com hipertensão arterial sistêmica.

compõe a Estratégia de Saúde da Família do Ministério da Saúde do Brasil, localizada na região urbana periférica da cidade de Belém - PA.

A casuística estudada foi composta por um corte de 33 pacientes (número obtido por cálculo de amostragem, determinado por um bioestatístico) com Hipertensão Arterial Sistêmica (HAS) inscritos no Programa Nacional de Atenção à Hipertensão Arterial e Diabetes Mellitus (HIPERDIA) da referida unidade, selecionados aleatoriamente, por meio de sorteio baseado nos números de matrícula realizado através de programa computacional por um bioestatístico especializado.

Utilizou-se como critério de inclusão para o sorteio: pacientes na faixa etária de 40 a 80 anos (faixa etária de maior prevalência de HAS e onde se concentram os maiores fatores de risco), de ambos os sexos e levando em consideração todas as classes sócio-econômicas. Os critérios para exclusão do sorteio foram: mulheres grávidas e os indivíduos que se recusassem a participar do estudo (responder ao questionário e/ou se submeter ao protocolo de pesquisa caso sorteados). Vale ressaltar que a taxa de exclusão foi de $0 \%$, tendo em vista que não havia pacientes gestantes dentro da faixa etária estabelecida para o estudo. Ademais, não houve recusa por parte dos pacientes em se submeter à pesquisa, fato este que foi atribuído às boas informações prestadas aos pacientes pelos pesquisadores e ao conteúdo esclarecedor do TCLE.

A Casa Saúde da Família Águas Lindas II funciona dentro do que é preconizado pela Estratégia de Saúde da Família e a captação dos pacientes (a maioria de baixa renda) é feita por meio de visitas domiciliares e triagem realizada pelos profissionais de saúde que a compõem. O HIPERDIA é um programa de cadastro e acompanhamento de pacientes hipertensos e/ou diabéticos que presta atendimentos médicos e de outras naturezas, bem como fornece medicação e orientações gratuitas aos pacientes cadastrados.

Foi aplicado um questionário de forma individual, em consulta agendada pelos pesquisadores na própria Casa Saúde da Família Águas Lindas II, após breve esclarecimento acerca das perguntas que o compõem. Os pesquisadores foram os responsáveis pela aplicação dos questionários, depois da padronização e treinamento de como as perguntas deveriam ser realizadas e como os pacientes deveriam ser abordados. Foram levantados dados referentes às condições sócio-econômicas e de outros aspectos que poderiam ter se configurado como fatores de risco no desenvolvimento da HAS dos estudados. As perguntas do questionário envolviam os seguintes pontos: idade (em anos), sexo, raça (auto-referida), renda salarial familiar (em reais), escolaridade, ocupação, tabagismo, etilismo, prática de exercício físico (mínimo de três vezes na semana por 30 minutos), antecedentes familiares de hipertensão arterial, co-morbidades e utilização ou não de medicação hipotensora.

Além do questionário, foram efetuadas verificações da pressão arterial (PA), do índice de massa corpórea (IMC) e da circunferência da cintura (CC) dos participantes, por meio de uma ficha de avaliação própria.

Para a aferição da pressão arterial (PA), foram seguidas as normas ditadas pela $V$ Diretrizes Brasileiras de Hipertensão Arterial ${ }^{4}$, utilizando-se um esfigmomanômetro aneróide da marca Diasyst ${ }^{\circledR}$ e um estetoscópio Littmann-Cardiology $\|^{\circledR}$. As aferições foram realizadas uma única vez no braço esquerdo. Considerou-se o início dos ruídos auscultatórios como a PA sistólica, e a extinção dos mesmos como a PA diastólica, ambas medidas em milímetros de mercúrio $(\mathrm{mmHg})$.

Com base nos dados coletados, foram considerados como valores pressóricos elevados os de pressão arterial sistólica (PAS) igual ou maior que 140 $\mathrm{mmHg}$ e/ou os de pressão arterial diastólica (PAD) igual ou maior que $90 \mathrm{mmHg}$, seguindo as orientações das Sociedades Brasileiras de Hipertensão, de Cardiologia e de Nefrologia ${ }^{8}$.

O índice de massa corpórea (IMC) foi calculado por meio da divisão do peso (em quilogramas) pelo quadrado da altura (em metros ao quadrado), sendo que tais medidas foram obtidas com o paciente em posição ortostática sobre uma balança antropométrica com estadiômetro da marca Fillizola ${ }^{\circledR}$. De acordo com os valores de IMC obtidos, os participantes do estudo foram classificados da seguinte forma, obedecendo ao que foi preconizado em 2000 pela Organização Mundial da Saúde (OMS): $<18,5 \mathrm{Kg} / \mathrm{m}^{2}$ (baixo peso); 18,5 a $24,9 \mathrm{Kg} / \mathrm{m}^{2}$ (peso normal); 25 a $29,9 \mathrm{Kg} / \mathrm{m}^{2}$ (sobrepeso); e $\geq 30 \mathrm{Kg} / \mathrm{m}^{2}$ (obesidade) ${ }^{9}$.

Para a medida da circunferência da cintura (CC) foi utilizada uma fita métrica inelástica, graduada em centímetros, posicionada ao redor da cicatriz umbilical (entre a crista ilíaca e o rebordo costal inferior). Os valores tidos como normais para a circunferência da cintura (CC) foram: abaixo de $80 \mathrm{~cm}$, para mulheres; e abaixo de $94 \mathrm{~cm}$, para homens. Valores acima dos indicados anteriormente foram considerados obesidade abdominal/ visceral/ centralizada, respeitando os parâmetros da Organização Mundial da Saúde (OMS) de $2000^{\circ}$.

Os dados obtidos foram analisados estatisticamente por meio do programa Bioestat ${ }^{\circledR}$ 4.0. De acordo com a natureza das variáveis, foi aplicada análise estatística descritiva, sendo informados os valores percentuais dos dados analisados. A confecção dos resultados em forma de gráficos e tabelas foi realizada por meio do software Microsoft ${ }^{\circledR}$ Excel 2007. 


\section{RESULTADOS}

TABELA 1. Caracterização biossocial dos pacientes hipertensos cadastrados no Programa HIPERDIA, na Casa Saúde da Família Águas Lindas II, Belém - PA em 2007

\begin{tabular}{|c|c|c|}
\hline Características & Freqüência & $\%$ \\
\hline \multicolumn{3}{|l|}{ Idade } \\
\hline 40 a 50 anos & 8 & 24 \\
\hline 51 a 60 anos & 5 & 15 \\
\hline 61 a 70 anos & 13 & 40 \\
\hline 71 a 80 anos & 7 & 21 \\
\hline Total & 33 & 100 \\
\hline \multicolumn{3}{|l|}{ Sexo } \\
\hline Feminino & 27 & 82 \\
\hline Masculino & 6 & 18 \\
\hline Total & 33 & 100 \\
\hline \multicolumn{3}{|l|}{ Raça } \\
\hline Branca & 5 & 15 \\
\hline Negra & 4 & 12 \\
\hline Mulata & 15 & 46 \\
\hline Mestiça & 8 & 24 \\
\hline Amarela & 1 & 3 \\
\hline Total & 33 & 100 \\
\hline \multicolumn{3}{|l|}{ Renda familiar } \\
\hline Menor que 1 salário & 6 & 18 \\
\hline Entre 1 e 2 salários & 20 & 61 \\
\hline Entre 2 e 3 salários & 7 & 21 \\
\hline Total & 33 & 100 \\
\hline \multicolumn{3}{|l|}{ Nível de escolaridade } \\
\hline Analfabeto & 8 & 24 \\
\hline Lê-escreve & 3 & 9 \\
\hline Fundamental incompleto & 17 & 52 \\
\hline Fundamental completo & 1 & 3 \\
\hline Médio incompleto & & 2 \\
\hline Médio completo & 1 & 3 \\
\hline Superior incompleto & 1 & 3 \\
\hline Total & 33 & 100 \\
\hline \multicolumn{3}{|l|}{ Ocupação } \\
\hline Do lar & 18 & 55 \\
\hline Aposentado(a) & 5 & 15 \\
\hline Outros & 10 & 30 \\
\hline Total & 33 & 100 \\
\hline
\end{tabular}

Fonte: Protocolo de pesquisa.
TABELA 2. Presença de tabagismo, etilismo, prática de exercício físico, antecedentes familiares, co-morbidades e uso de medicação hipotensora por parte dos pacientes hipertensos cadastrados no Programa HIPERDIA, na Casa Saúde da Família Águas Lindas II, Belém - PA em 2007

\begin{tabular}{|c|c|c|}
\hline Características & Freqüência & $\%$ \\
\hline \multicolumn{3}{|l|}{ Tabagismo } \\
\hline Sim & 2 & 6 \\
\hline Não & 31 & 94 \\
\hline Total & 33 & 100 \\
\hline \multicolumn{3}{|l|}{ Etilismo } \\
\hline Sim & 5 & 15 \\
\hline Não & 28 & 85 \\
\hline Total & 33 & 100 \\
\hline \multicolumn{3}{|l|}{ Exercício físico } \\
\hline Sim & 12 & 36 \\
\hline Não & 21 & 64 \\
\hline Total & 33 & 100 \\
\hline \multicolumn{3}{|c|}{ Antecedendes familiares } \\
\hline Sim & 16 & 48 \\
\hline Não & 17 & 52 \\
\hline Total & 33 & 100 \\
\hline \multicolumn{3}{|l|}{ Co-morbidades } \\
\hline Sim & 13 & 39 \\
\hline Não & 20 & 61 \\
\hline Total & 33 & 100 \\
\hline \multicolumn{3}{|c|}{ Medicação hipotensora } \\
\hline Sim & 33 & 100 \\
\hline Não & 0 & 0 \\
\hline Total & 33 & 100 \\
\hline
\end{tabular}

Fonte: Protocolo de pesquisa. 
Dias EM, et al. Perfil epidemiológico dos pacientes com hipertensão arterial sistêmica.

GRÁFICO 1. Distribuição, de acordo com o controle da pressão arterial sistólica (PAS) e da pressão arterial diastólica (PAD), dos pacientes hipertensos cadastrados no Programa HIPERDIA, na Casa Saúde da Família Águas Lindas II, Belém - PA em 2007

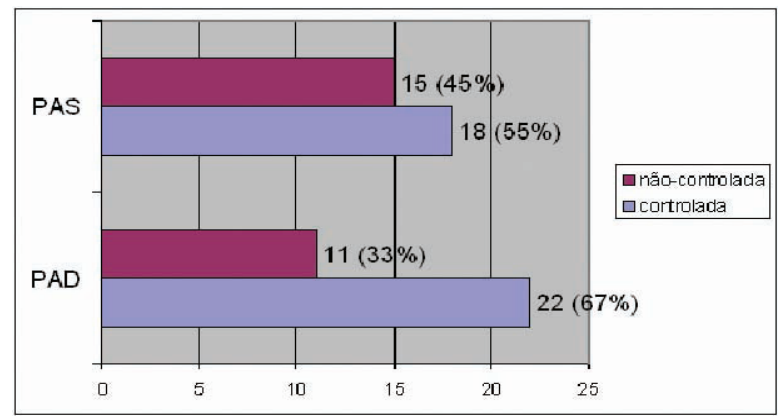

GRÁFICO 2. Distribuição, de acordo com a classificação do índice de massa corpórea (IMC), dos pacientes hipertensos cadastrados no Programa HIPERDIA, na Casa Saúde da Família Águas Lindas II, Belém - PA em 2007

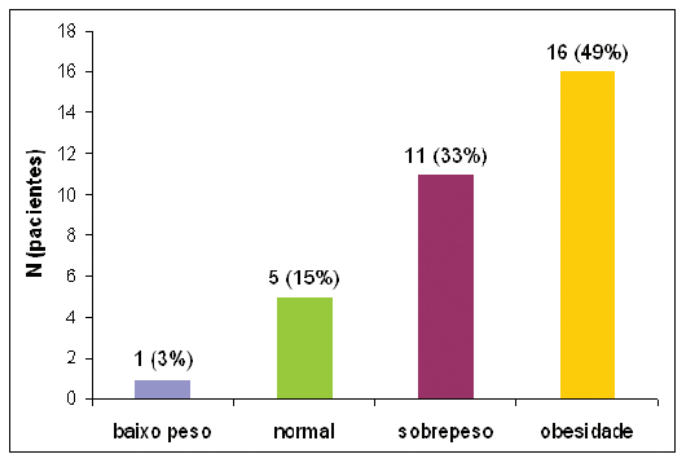

GRÁFICO 3. Distribuição, de acordo com a classificação da medida de circunferência da cintura, dos pacientes hipertensos cadastrados no Programa HIPERDIA, na Casa Saúde da Família Águas Lindas II, Belém - PA em 2007

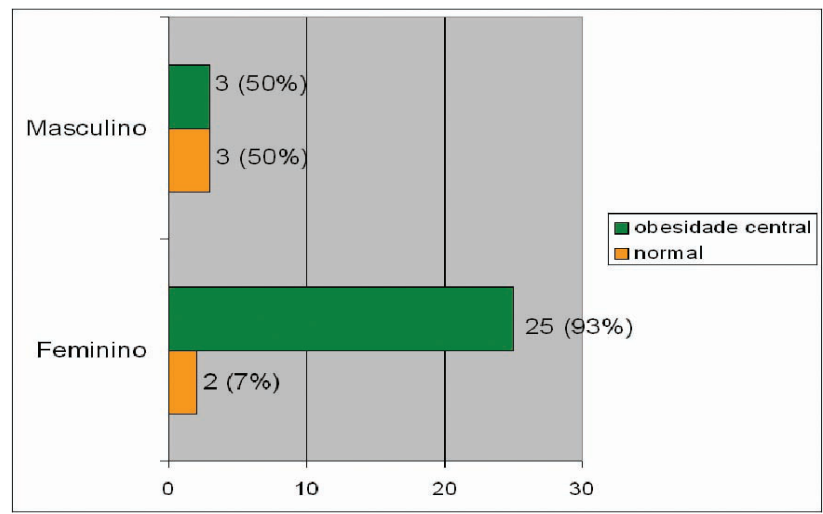

\section{DISCUSSÃO}

São inúmeros os fatores que determinam e/ ou influenciam o desenvolvimento da HAS, alguns inerentes ao indivíduo (idade, sexo e raça), outros relacionados ao estilo de vida (tabagismo, etilismo, sedentarismo, etc.) e ainda outras condições de risco.

Há, naturalmente, com o avançar da idade, redução da complacência dos vasos, enrijecimento das artérias, e diminuição de sua elasticidade, entre outros aspectos fisiopatológicos que comprometem a boa função cardíaca e contribuem de forma importante para o surgimento de $\mathrm{HAS}^{2,10}$.

Esse fato é comprovado em estudos epidemiológicos, como o de Monteiro et al. ${ }^{3}$, em que a maior prevalência da doença ocorreu em pessoas mais velhas; o que também foi observado pelo presente estudo, estando a maioria na faixa etária de 61 a 70 anos (Tabela 1).

Até os 40 anos, os homens são os mais atingidos pela HAS; contudo, após a menopausa, o ganho de peso e as alterações hormonais possivelmente explicam o aumento da PA nas mulheres $e$, consequentemente, a prevalência da HAS nessas em relação aos homens a partir de então 2,10 . Essa predominância da doença no sexo feminino pode ser observada no estudo em questão (Tabela I), bem como nas pesquisas de Sodré et al. ${ }^{11}$ e Boing e Boing ${ }^{6}$.

Ainda se poderia admitir, como explicação para o maior índice de mulheres hipertensas inscritas no Programa de Hipertensão (HIPERDIA), a questão das mesmas serem mais ativas do que os homens no que se refere à procura por orientações e tratamentos nos postos de saúde ${ }^{6,12}$.

Quanto à raça, a literatura relata que a prevalência de HAS é duas vezes maior em negros do que em brancos ${ }^{13}$. Estudos realizados com populações afro-americanas sugeriram que a maior prevalência e gravidade da HAS em negros pode ser explicada pelos maiores níveis de vasopressina plasmática e declínio da resposta renal neste grupo étnico ${ }^{2}$.

Contudo, foram observadas variações nas pesquisas de Trindade et al. ${ }^{14}$, Converso e Leocádio ${ }^{15}$ e Mano e Pierin ${ }^{16}$, nas quais houve predominância de hipertensos da raça branca. Já neste estudo, verificou-se um maior número de mulatos e mestiços com a doença, o que pode ser explicado pelo alto grau de miscigenação na região onde o mesmo foi realizado (Tabela 1 ).

A condição sócio-econômica também pode ser citada como fator de influência para $\mathrm{HAS}^{17}$, que pode ser analisada, neste caso, pelo nível de escolaridade, renda salarial e profissão/ocupação.

A baixa escolaridade dos pacientes pode ser um fator dificultador para a melhor adesão ao tratamento da $\mathrm{HAS}^{16}$, já que essa interfere 
negativamente na assimilação das orientações necessárias ao tratamento ${ }^{10}$.

Constatou-se que mais da metade dos indivíduos hipertensos não tinham ao menos o ensino fundamental completo (Tabela 1). Resultados semelhantes foram observados nos estudos de Simonetti et al. ${ }^{18}$ e Monteiro et al. ${ }^{3}$.

Sousa et al. ${ }^{19}$ verificaram, além do baixo grau de formação escolar dos hipertensos de sua pesquisa, que a renda salarial familiar da maioria desses não ultrapassava dois salários mínimos. Esse perfil também pode ser observado no presente estudo (Tabela 1).

O baixo poder aquisitivo indica a importância da assistência do Sistema Único de Saúde (SUS) aos portadores de HAS, haja vista que esses não têm como se valer de planos particulares ${ }^{19}$.

Ademais, pesquisas relacionam ocupação ao aumento da pressão arterial, sendo que quanto menor é o status ocupacional, maior é a tendência de aumento da pressão arterial. E isso pode ser explicado pela presença de várias situações agravantes, como insatisfação no trabalho, estresse ambiental e social etc., que alteram os níveis pressóricos ${ }^{10,20}$.

Na presente pesquisa, as tarefas do lar foram a ocupação predominante (Tabela 1), haja vista que o grupo feminino prevaleceu, resultado esse que é compartilhado por Monteiro et al. ${ }^{3}$.

Quanto aos hábitos dos indivíduos, podem-se mencionar o tabagismo e o etilismo como dois fatores de risco com grande influência no desenvolvimento da HAS e no aumento do risco de morte cardiovascular. Além disso, o uso de cigarros e álcool pelos hipertensos causa tolerância à ação anti-hipertensiva dos medicamentos ${ }^{16}$.

A nicotina é prejudicial ao organismo por promover a liberação de catecolaminas, que aumentam a frequência cardíaca, a pressão arterial e a resistência periférica. Além disso, a nicotina facilita a deposição de gordura nos vasos, devido à lesão que essa provoca nos mesmos ${ }^{10}$.

O álcool, semelhantemente, quando em taxas aumentadas no sangue, eleva a pressão arterial lenta e progressivamente, na proporção de $2 \mathrm{mmHg}$ para cada $30 \mathrm{ml}$ de álcool ingeridos diariamente ${ }^{10}$.

O tabagismo e o etilismo/alcoolismo não estiveram presentes em grande proporção nos estudos com hipertensos de Mano e Pierin ${ }^{16}$ e Simonetti et al. ${ }^{18}$, bem como no estudo em questão (Tabela 2 ).

É sabido que a prática de exercício físico traz inúmeros benefícios à saúde e possui efeitos hipotensores, importantes no caso dos indivíduos hipertensos ${ }^{17}$. Esse fato pode ser verificado na pesquisa de Souza et al. ${ }^{21}$, com idosos hipertensos que, após quatro semanas de exercícios físicos supervi- sionados, tiveram seus níveis pressóricos diminuídos significativamente.

Este mecanismo hipotensor do exercício físico é decorrente de efeitos hemodinâmicos e neurais. Os primeiros incluem: diminuição da resistência vascular periférica total e do débito cardíaco, proveniente da redução da frequência cardíaca e do volume sistólico. Os efeitos neurais: diminuição da atividade nervosa simpática, reduzindo os níveis de norepinefrina. $\mathrm{E}$ ainda, aumento das vias antioxidantes na vasculatura e redução de LDL oxidado ${ }^{21}$.

Em estudos como os de Monteiro et al. ${ }^{3} \mathrm{e}$ de Boing e Boing ${ }^{6}$ foi observado um alto índice de hipertensos sedentários, assim como na presente pesquisa (Tabela 2), sendo que a justificativa dos pacientes para a não execução de exercício físico, em geral, foi a falta de disposição e de tempo devido ao trabalho e, ainda, a idade avançada.

Além dos fatores de risco modificáveis, é de conhecimento geral que a HAS possui um fator que independe dos hábitos do indivíduo: a presença de carga genética favorável ao desenvolvimento da doença.

A possibilidade de um indivíduo desenvolver HAS é muito maior se o mesmo tiver história familiar da doença ${ }^{2,15}$.

Converso e Leocádio ${ }^{15}$ e Simonetti et al. ${ }^{18}$ verificaram número expressivo de hipertensos com fator genético para a doença, sendo que no presente estudo, houve equivalência entre presença e ausência de história familiar nos participantes da pesquisa (Tabela 2).

A obesidade, seja centralizada ou generalizada, é um dos fatores de risco de maior relevância para o desenvolvimento de HAS e outras patologias cardiovasculares ${ }^{22,23}$. Tal relação entre a adiposidade corporal e a elevada prevalência de HAS foi constatada nos estudos de Carneiro et al. ${ }^{23}$ e de Cabrera e Jacob Filho ${ }^{24}$.

Visando a detectar a presença desse fator de risco em estudos epidemiológicos, são utilizadas as medidas antropométricas do Índice de Massa Corpórea (IMC) e Circunferência da Cintura (CC), que representam uma maneira racional e eficiente de se presumir o volume e a distribuição de gordura, além de serem de fácil aplicação e baixo custo ${ }^{24,25}$.

O IMC é tido como um bom preditor de obesidade generalizada, ao passo que a CC detecta a obesidade visceral ou centralizada, que tem forte relação com doenças metabólicas e coronarianas ${ }^{26}$.

A pesquisa em questão, com base nessas medidas antropométricas, constatou que um número expressivo dos hipertensos estudados apresentou sobrepeso/obesidade generalizada e visceral (Gráficos 2 e 3). 
Dias EM, et al. Perfil epidemiológico dos pacientes com hipertensão arterial sistêmica.

É importante notar que um número significativo de indivíduos apresentou PAS e PAD elevadas/não controladas (Gráfico 1), mesmo estando todos os pacientes da amostra em uso de medicação hipotensora (Tabela 2), obedecendo ao esquema terapêutico preconizado pelo programa HIPERDIA do Ministério da Saúde.

Monteiro et al. ${ }^{3}$, Mano e Pierin ${ }^{16}$ e Sousa et al. ${ }^{19}$ também observaram esse mesmo quadro em suas pesquisas com pacientes hipertensos acompanhados por programas de controle da hipertensão. Os mesmos argumentam que tal fato se deve à falta de adesão por parte desses aos esquemas terapêuticos e à orientação inadequada fornecida pelos profissionais de saúde.

Deve-se lembrar que a hipertensão dificilmente existe como um problema isolado, mas está acompanhada de outras doenças relacionadas, que podem tanto ser conseqüência da PA elevada quanto da ação conjunta dos fatores desencadeadores da $\mathrm{HAS}^{1,3,6}$

Boing e Boing ${ }^{6}$, em seu estudo junto ao Sistema de Informações Hospitalares (SIH) - o qual registra as internações hospitalares na rede SUS -, verificaram altas taxas de internações por Acidente Vascular Encefálico (AVE) e Insuficiência Cardíaca Congestiva (ICC) em todas as regiões do Brasil, resultado dos níveis pressóricos não-controlados desses indivíduos.

Simonetti et al..$^{18}$, em sua pesquisa, verificaram que a totalidade de indivíduos estudados apresentava algum tipo de comprometimento dos órgãos-alvo. Nesse estudo, a ocorrência de co-morbidades foi de aproximadamente $40 \%$ dos pacientes (Tabela 2 ).

A detecção e o tratamento precoce da HAS tornam-se necessários para evitar o aparecimento de tais co-morbidades.

\section{CONCLUSÃO}

O perfil epidemiológico dos pacientes foi caracterizado por: predominância do sexo feminino e faixa etária de 61 a 70 anos; mulatos; baixo nível de escolaridade e de renda salarial; baixa ocorrência de etilismo, tabagismo e prática de exercício físico; baixo índice de co-morbidades e antecedentes familiares; e alta ocorrência de sobrepeso e obesidade. Os níveis tensionais se apresentaram controlados na maioria dos pacientes, embora um número significativo desses estivesse com a pressão arterial (PA) descontrolada.

Estudos epidemiológicos como esse, sinalizam para a importância de se instituir programas de saúde pública que visem a combater os fatores de risco envolvidos com a gênese e complicações da HAS.

Dias EM, Pereira RMN, Cavalcante HS, Ramalheiro NS, Silva NF, Carvalho Júnior MR. Epidemiological profile of patients with systemic arterial hypertension, registered in the Casa Saúde da Família Águas Lindas II, Belém - PA. Rev Med (São Paulo). 2009 jul.-dez.;88(3/4):191-8.

ABSTRACT: OBJECTIVE: Defining the epidemiological profile of the patients with Systemic Arterial Hypertension(SAH) registered in Programa Nacional de Atenção à Hipertensão Arterial e Diabetes Mellitus (HIPERDIA) of Casa Saúde da Família Águas Lindas II, in Belém, Pará, in 2007. METHODS: An observational, descriptive, cross-sectional study in a sample of 33 people aged 40 to 80 years, both genders, who were registered in HIPERDIA of that health center and selected randomly using their registration number. A questionnaire on biosocial characteristics and life habits was applied. The values for Blood Pressure (aneroid sphygmomanometer Diasyst ${ }^{\circledR}$ and stethoscope Littmann-Cardiology ${ }^{\circledR}{ }^{\circledR}$ ), Body Mass Index (anthropometric balance with stadiometer Fillizola ${ }^{\circledR}$ ) and Waist Circunference (inelastic tape measure) were registered. A descriptive statistical analysis was conducted using the Bioestat ${ }^{\circledR} 4.0$ and the Microsoft ${ }^{\circledR}$ Excel 2007 programs. RESULTS: It was observed that the majority (40\%) of the patients aged 61 to 70 years, $82 \%$ were women; race "mulata" (46\%); wage income of 1 to 2 minimum wages $(61 \%)$; incomplete elementary school (52\%), occupation "home care" $(55 \%)$; majority of non-smokers $(94 \%)$, non-drinkers $(85 \%)$, sedentary $(64 \%)$, no familiar antecedent of SAH $(52 \%)$; no comorbidities $(61 \%)$; all the patients have used hypotensive medicine; pressure level controlled in the majority of the patients (systolic blood pressure - 55\%; diastolic blood pressure - 67\%); high percentage of general obesity and/or overweight (82\%) and central obesity (men-50\%; women -93\%). CONCLUSION: The studied sample was characterized predominantly by: women; aged 61 to 70 years; "mulatos"; low schooling level and wage income; low rate of: alcoholism, smoking, practice of physical exercise, comorbidities and familiar antecedents of the disease; high rate of overweight/obesity; pressure level controlled, although a high number of patients had the blood pressure uncontrolled.

KEY WORDS: Hypertension/epidemiology; Blood pressure; Obesity. 


\section{REFERÊNCIAS}

1. Brasil. Ministério da Saúde. Secretaria de Atenção à Saúde. Departamento de Atenção Básica. Hipertensão arterial sistêmica para o Sistema Único de Saúde. Brasília, DF; 2006.

2. Amado TCF, Arruda IKG. Hipertensão arterial no idoso e fatores de risco associados. Rev Bras Nutr Clin. 2004;19(2):94-9.

3. Monteiro PC, Santos FS, Fornazari PA, Cesarino CB Características biossociais, hábitos de vida e controle da pressão arterial dos pacientes em um programa de hipertensão. Arq Ciênc Saúde. 2005;12(2):73-9.

4. Sociedade Brasileira de Hipertensão. V Diretrizes Brasileiras de Hipertensão Arterial. Rev Bras Hipertens. 2006;13(4):256-312.

5. Lemos DM, Fundão LN, Ferreira MVL, Mill JG. Redução quantitativa do risco cardiovascular no tratamento da hipertensão arterial em unidade do Programa de Saúde da Família. Rev Bras Hipertens. 2006;13(2):117-25.

6. Boing $A C$, Boing AF. Hipertensão arterial sistêmica: o que nos dizem os sistemas brasileiros de cadastramentos e informações em saúde. Rev Bras Hipertens. 2007;14(2):84-8.

7. Palmeira MM, Leão WC, Marinho EM, Ferreira TPS, Santos JPC, Sousa FCS, et al. Perfil epidemiológico dos pacientes hipertensos e seus principais fatores de risco para doenças cardiovasculares, no Ambulatório de Cardiologia da Universidade do Estado do Pará. In: 17ํㅡㄹ Congresso Paraense de Cardiologia, Belém, Pará, out. 2007. Anais. SBC/PA; 2007. p.30 [Resumo, 003].

8. Sociedade Brasileira de Hipertensão, Sociedade Brasileira de Cardiologia e Sociedade Brasileira de Nefrologia. IV Diretrizes Brasileiras de Hipertensão Arterial. Rev Arq Bras Cardiol. 2004;82(supl. 4):1-4.

9. World Health Organization. Obesity: preventing and managing the global epidemic. Report. Geneva; 2000.

10. Pessuto J, Carvalho EC. Fatores de risco em indivíduos com hipertensão arterial. Rev Latinoamer Enf. 1998;6(1):33-9.

11. Sodré EFLM, Borges MMG, Macedo LFC, Pereira AM, Pacheco MP. Perfil epidemiológico de hipertensos cadastrados na Casa Família Água Cristal em Belém - PA. In: 16을 Congresso Paraense de Cardiologia, Belém, PA, 2006. Anais. SBC/PA; 2006. p.33 [Resumo, 002].

12. Almeida MF, Barata RB, Montero CV. Prevalência de doenças crônicas auto-referidas e utilização de serviços de saúde. Rev Ciên Saúde Coletiva. 2002;7(4):743-56.

13. Porto CC. Hipertensão arterial sistêmica - hábitos de vida e fatores correlatos. JBM. 1999;76:35-45.

14. Trindade IS, Heineck G, Machado JR, Ayzemberg H, Formighieri M, Crestani M, et al. Prevalência da hipertensão arterial sistêmica na população urbana de Passo Fundo (RS). Arq Bras Cardiol. 1998;71(2):127-30.
15. Converso MER, Leocádio PLL. Prevalência da hipertensão arterial e análise de seus fatores de risco em idosos de Presidente Prudente. In: $2^{\circ}$ Congresso Brasileiro de Extensão Universitária, Belo Horizonte, MG, set. 2004. Anais. UESP; 2004.

16. Mano GMP, Pierin AMG. Avaliação de pacientes hipertensos acompanhados pelo Programa Saúde da Família em um Centro de Saúde Escola. Acta Paul Enferm. 2005;18(3):269-75.

17. Lima MT, Bucher JSNF, Lima JWO. A hipertensão arterial sob o olhar de uma população carente: estudo exploratório a partir dos conhecimentos, atitudes e práticas. Cad Saúde Pública. 2004;20(4):1079-87.

18. Simonetti JP, Batista L, Carvalho LR. Hábitos de saúde e fatores de risco em pacientes hipertensos. Rev Latinoam Enf. 2002;10(3):415-22.

19. Sousa LB, Souza RKT, Scochi MJ. Hipertensão arterial e saúde da família: atenção aos portadores em município de pequeno porte na região sul do Brasil. Arq Bras Cardiol. 2006;87(4):496-503.

20. Cordeiro R, Fischer FM, Lima Filho EC, Moreira Filho DC. Ocupação e hipertensão. Rev Saúde Pública. 1993;27(5):380-7.

21. Souza PFP, Cavalcante BV, Silva HC, Yokote Filho M, Costa WJM. Efeitos do exercício físico supervisionado em hipertensos idosos. Rev Pa Cardiol. 2007;13(1):6-7.

22. Ribeiro Filho FSF, Rosa EC, Faria NA, Larário DDG, Ferreira SRG, Kohlmann O, et al. Obesidade, hipertensão arterial e suas influências sobre a massa e função do ventrículo esquerdo. Arq Bras Endocrinol Metab. 2000;44(1):64-71.

23. Carneiro G, Faria NA, Ribeiro Filho FF, Guimarães $A$, Lerário D, Ferreira SRG, et al. Influência da distribuição da gordura corporal sobre a prevalência de hipertensão arterial e outros fatores de risco cardiovascular em indivíduos obesos. Rev Assoc Med Bras. 2003;49(3):306-11.

24. Cabrera MAS, Jacob Filho W. Obesidade em idosos: prevalência, distribuição e associação com hábitos e co-morbidades. Arq Bras Endocrinol Metab. 2001;45(5):494-501.

25. Dias EM, Oliveira PB, Pereira RMN, Tostes CL, Costa DT. Identificação de correlação entre índice de massa corpórea (IMC) e percentual de gordura (\%G) em funcionários da UNAMA visitantes da SIPAT - 2006. In: $17^{\circ}$ Congresso Paraense de Cardiologia, Belém, Para, out. 2007. Anais. SBC/PA; 2007. p.29 [Resumo 005].

26. Martins IS, Marinho SP. O potencial diagnóstico dos indicadores da obesidade centralizada. Rev Saúde Pública. 2003;37(6):760-67.

Recebido para publicação em: 09/08/2009

Aceito para publicação em: 20/12/2009 